\title{
Phase formation in undercooled Sm-Co alloy melts
}

\author{
S. Reutzel ${ }^{\mathrm{a}, \mathrm{b}, *}$, J. Strohmenger ${ }^{\mathrm{b}}$, T. Volkmann ${ }^{\mathrm{b}}$, J. Gao $^{\mathrm{c}}$, D.M. Herlach ${ }^{\mathrm{b}}$ \\ ${ }^{a}$ Institute of Experimental Physics IV, Ruhr-University, 44780 Bochum, Germany \\ ${ }^{\mathrm{b}}$ Institute of Space Simulation, German Aerospace Centre (DLR), 51170 Cologne, Germany \\ ${ }^{c}$ Key Laboratory of Electromagnetic Processing of Materials, Northeastern University, Shenyang 110004, China
}

Received 21 August 2005; received in revised form 15 November 2005; accepted 15 February 2006

\begin{abstract}
$\mathrm{Sm}_{x} \mathrm{Co}_{100-x}$ alloy melts $(x=10.5,12.5$ and 17 at.\%) are processed containerlessly by electromagnetic levitation and drop-tube technique in order to get access to the metastable state of the undercooled melt. By dint of undercooling non-equilibrium phase formation is facilitated. The solidified specimen are subsequently analyzed by electron microscopy, X-ray diffraction and thermal magnetic analyses. The results show that different types of solidification behaviors are observed depending on alloy composition and undercooling level prior to solidification. Bulk samples processed by electromagnetic levitation technique are solidified by primary formation of either $\mathrm{Sm}_{2} \mathrm{Co}_{17}$ or $\mathrm{SmCo}_{5}$, whereas primary phase formation of metastable $\mathrm{SmCo}_{7}$ is detected in small particles attained by drop-tube processing where cooling rates lie in the order of $10^{3}-10^{5} \mathrm{~K} / \mathrm{s}$.
\end{abstract}

(C) 2006 Elsevier B.V. All rights reserved.

Keywords: Transition metal alloys; Rare earth alloys; Intermetallic compounds; Rapid solidification; Metastable phase formation

\section{Introduction}

Sm-Co alloys are important rare earth-transition metal magnetic materials for high temperature applications. In Co-rich composition, two equilibrium phases are formed which determine the properties of traditional permanent magnet materials: $\mathrm{SmCo}_{5}$ of the hexagonal $\mathrm{CaCu}_{5}$-type structure and $\mathrm{Sm}_{2} \mathrm{Co}_{17}$ of the rhombohedral $\mathrm{Th}_{2} \mathrm{Zn}_{17}$-type structure. The rhombohedral $\mathrm{Sm}_{2} \mathrm{Co}_{17}$ is related to hexagonal $\mathrm{SmCo}_{5}$ through the ordered substitution of Sm by a pair of Co atoms (Co dumbbells) resulting in correlated unit cell parameters. Under non-equilibrium solidification a metastable phase, $\mathrm{SmCo}_{7}$, is found $[1,2]$ which has the same unit cell as the $\mathrm{CaCu}_{5}$ structure, but a randomized (disordered) arrangement of Co dumbbells on the rare earth lattice sites, and hence different lattice parameters. This disordered arrangement belongs to the $\mathrm{TbCu}_{7}$-type structure [3] .

$\mathrm{SmCo}_{7}$ is reported to exhibit a lower temperature coefficient of intrinsic coercitivity than that of the stable phases [1,2,4,5], and is hence of high interest for application at elevated temperatures. In this work, it is studied how undercooling level and cooling rate enable the metastable phase formation of $\mathrm{SmCo}_{7}$

\footnotetext{
* Corresponding author. Tel.: +49 2203601 3047; fax: +49 22036012255 . E-mail address: sven.reutzel@ dlr.de (S. Reutzel).
}

and influence the microstructure of the solidified Sm-Co alloy specimen.

\section{Experimental}

Alloys with composition of $\mathrm{Sm}_{10.5} \mathrm{Co}_{89.5}, \mathrm{Sm}_{12.5} \mathrm{Co}_{87.5}$ and $\mathrm{Sm}_{17} \mathrm{Co}_{83}$ (at.\%) were prepared by arc-melting high purity elemental materials (Co 99.995\% and Sm 99.9\%) under the protection of an argon atmosphere (99.999\% purity). Considering that levitated samples were usually subjected to a loss of elemental Sm during overheating, the effect of changing alloy composition on the formation of $\mathrm{SmCo}_{7}$ should be considered. For this reason, an excess amount of $10 \% \mathrm{Sm}$ was added to the three base compositions, $\mathrm{Sm}_{10.5} \mathrm{Co}_{89.5}, \mathrm{Sm}_{12.5} \mathrm{Co}_{87.5}$ and $\mathrm{Sm}_{17} \mathrm{Co}_{83}$.

Before levitation, an alloy sample of about $1.2 \mathrm{~g}$ was placed onto a hollow quartz sample holder and positioned in the levitation coil of an electromagnetic levitation facility, of which the details can be found elsewhere [6]. After evacuation to a pressure in the order of $10^{-6} \mathrm{mbar}$, the chamber of the levitation facility was backfilled with highly purified helium gas (99.9999\%) to a pressure of about 500 mbar. The alloy sample was then levitated and melted under this pressure. In order to attain a substantial undercooling, the sample was shortly overheated to a temperature of 100-200 K above liquidus temperature. Subsequently, the sample was cooled by blowing helium gas onto the 